\title{
MONETARY UNIT SAMPLING USING MSEXCEL - AUTOMATION IN EXTERNAL AUDITING
}

\author{
Mihailo A. Todorovićn ${ }^{1,2 *}$, \\ Miroslav Ž. Perić \\ ${ }^{1} \mathrm{PhD}$ candidate, \\ Singidunum University, \\ Belgrade, Serbia \\ ${ }^{2}$ Singidunum University, \\ Belgrade, Serbia
}

\begin{abstract}
:
In the preparatory phase of auditing the client, a proper assessment of materiality is necessary, since it determines the scope of population testing. Furthermore, determining the proper model and the type of population sampling ensures that the real business of the entity will be shown in the financial statements. Depending on their size, audit companies use different tools in their work. The largest audit companies ("Big Four") use expensive software, while small and medium-sized audit companies use tools that they can develop or acquire, depending on their capabilities. In this paper, we discuss the viability of small and medium companies using Excel to implement features present in General auditing software. Two processes - stratification and monetary unit sampling are provided as examples.
\end{abstract}

Keywords:

external audit, CAATT, MSExcel, Monetary-Unit Sampling, stratification.

\section{INTRODUCTION}

Auditors rely on a variety of computer assisted auditing tools and techniques (CAATT), whose purpose is to increase the processing speed, reduce errors and improve operating efficiency (Lin C.-W., Wang C.-H. 2011.). The software and tools, which CAATT comprise of, can be further categorized into two groups:

1. General auditing software (GAS) such as Audit command language (ACL), or Caseware's Interactive data extraction and analysis (IDEA). These tools are considered to be professional and offer a wide range of utility.

2. Complementary software such as Excel or Access. These tools are more commonly used as they are less complex to implement in comparison to GAS, which is why they are preferred in smaller and medium sized auditing companies.

The choice between which tools to use is entirely up to the company and their available resources, as the International standards on auditing (ISA) do not officially proclaim a specific software for auditing purposes. While larger auditing companies and the „Big Four" have resources to either develop their own tools internally or purchase a custom made software which caters to their specific needs (adapted to the audit firm's group methodology and used by the whole group anywhere in the world), small and medium sized companies are left with a choice between investing into GAS and using complementary software to compensate for certain

Correspondence: Mihailo A. Todorović

e-mail: mtodorovic@singidunum.ac.rs tasks when necessary, or exclusively using complementary software for auditing.

A study in the United Kingdom found that a majority of small and medium sized external auditing companies in the UK made no use of GAS at all, stating that they found high implementation costs, significant learning curves and 
adoption processes off-putting, instead voicing their preference of traditional manual auditing methods. (Ahmi A., Kent S. 2012.)

In this paper we investigate whether an auditor can create a tool using complementary software that can offer a similar level of automation that GAS offers. As an example, we provide a tool for stratification and assistance in selection when using monetary unit sampling made in MSExcel using Visual Basic for Applications (VBA).

\section{LITERATURE REVIEW}

In a research paper written by Ching-Wen Lin and Chih-Hung Wang (2011), different types of CAATTs are discussed and main decision-making factors that need to be considered when assessing auditing software are listed. Lin and Wang use a focus group interview and analytic network process (ANP) to develop their model for assessment of auditing software. The authors use Audit Command Language (ACL), Interactive data extraction and analysis (IDEA) and Focaudit as the assessment examples and note the differences between user experiences with them.

Aidi Ahmi and Simon Kent (2012) discuss the lack of utilization of generalized audit software by external auditors in the United Kingdom. Ahmi and Kent used a webbased survey to gather the perceptions of 205 statutory auditors in the UK. The authors stated that $73 \%$ of external auditors made no use of GAS, and discovered which particular factors influence the likelihood of auditors using GAS, as opposed to the traditional auditing methods.

The International Auditing and Assurance Standards Board (IAASB), which sets high-quality international standards for auditing, assurance and quality control, provides the standard for sampling in auditing - ISA 530. ISA 530 describes the process of sampling and the principal sample selection methods.

An online article for the CPA Journal written by Bruce Wampler and Michelle McEacharn (2005) proposes an automated solution for Monetary-Unit Sampling (MUS) made in MSExcel, along with instructions and examples of how to determine the sample size, select the sample and evaluate the results.

American Institute of Certified Public Accountants (AICPA) offers a guideline on audit sampling in their publication "Audit Guide: Audit Sampling” (2014). Chapter 6 of the Audit Guide which will later be referenced explains in detail the characteristics of MUS, how to define the sample unit, select the sample and determine the sample size when using MUS, as well as an example with an evaluation of the sample size.

The foregoing literature will assist with defining the terminology in the upcoming sections, along with the offered insight into different publications regarding this topic.
Upon reviewing the literature, it can be concluded that aside from Bruce Wampler and Michelle McEacharn there are not many publications that discuss the topic of auditors building MUS in Excel.

\section{METHODOLOGY}

In this section, audit sampling and stratification will be defined, as well as the approach an auditor should take when creating tools.

Audit sampling is defined as the appliation of audit procedures to less than $100 \%$ of items within a population of audit relevance such that all sampling units have a chance of selection in order to provide the auditor with a reasonable basis on which to draw conclusions about the entire population (ISA 530, IAASB Handbook, 2018).

Stratification is defined as the process of dividing a population into sub-populations, each of which is a group of sampling unit which have similar characteristics (often monetary value) (ISA 530, IAASB Handbook, 2018).

In audit, sampling is used when an auditor, due to the nature of the transactions concludes that it is not necessary to test all of the transactions in the population, or when testing all of the transactions would require a large amount of time and resources, and therefore would be impractical and uneconomical. (Mijoč I., Mijoč J., 2011)

Mijoč and Mijoč also state the advantages and disadvantages of using sampling in audit. Lower costs, faster processing of data and results, flexibility and reliability are listed as advantages; while the lack of data for each unit in the sample and the additional employee training required adequately choosing a sample and extrapolating the misstatements (over-statements and under-statements), are listed as disadvantages of using this method.

The principal sampling methods stated in the ISA 530 include random selection, systematic selection, haphazard selection (in the case of non-statistical sampling), block selection and monetary unit sampling. Most of these sampling methods are already implemented in GAS and can be implemented in Excel as well through various plug-ins available online.

As it was previously mentioned, the tool which is discussed in this paper is an example of monetary unit sampling. ISA 530 describes MUS as a type of value-weighted selection in which sample size, selection, and evaluation results in a conclusion in monetary amounts. The aforementioned leads to the conclusion that MUS is a type of attribute sampling. Attribute sampling is typically used to reach a conclusion about a population in terms of a rate of occurrence. MUS expresses this conclusion in dollar amounts instead of the rate of occurrence. 
MUS is also a subset of a broader class of procedures referred to as probability proportional to size (PPS) sampling. The characteristic of PPS samples is that the probability of an item's selection is proportional to its recorded amount.

One of the advantages of using MUS would be that it is generally easier to apply than classical variables sampling, since it is based on attribute sampling theory and the auditor can easily calculate sample sizes and evaluate their results using a computer program (such as the provided example) or a calculator. It also does not require direct consideration of the population characteristics, and usually results in a highly efficient sample size.

MUS is especially useful when it comes to accounts receivable confirmation, loans receivable confirmation, tests of investment security pricing compared to published prices, inventory price tests in which the auditor anticipates less misstatements and the population does not contain a number of large understatements and in fixed-asset additions tests where existence is the primary risk.

One of the disadvantages of using MUS would be that it is not designed to test understatements of a population. Since the sample is selected proportional to size, it is less likely that many of the small recorded amounts will be selected, yet those units can potentially be significantly understated. Another disadvantage would be that if the expected amount of misstatements increases, the appropriate MUS sample size will also increase. In these cases, it might be more appropriate to use other sampling techniques (AICPA, Audit Guide: Audit Sampling, 2014).

In the process of creating a tool in Excel, or any other software for the purpose of auditing, auditors should consider the following:

1. The tool is universally applicable. Since the topic of this paper are tools created by small and medium sized auditing companies, it would be a large investment for them to create a solution that can only be applied to one specific scenario each time. Rather, the auditors should focus on creating a tool that offers a solution for multiple clients, with minor modifications. In the case of monetary unit sampling and stratification, an example could be that the tool performs equally regardless of the population size.

2. The tool has instructions built in it. This reduces the learning curve for employees and minimizes the chance of them making a mistake in the process. In the case of monetary unit sampling and stratification, an example of an instruction could be where to import the population, or whether the format of the imported population should be in a certain order.
3. The auditor should be able to explain the purpose and the results of the tool to Quality Control. With well-defined instructions and adequate understanding of the tool, quality control should come to the conclusion that the tool is appropriate for use and offers relevant results.

The example of the aforementioned MUS coded in Excel will be presented in the upcoming section.

\section{RESULTS}

In this section, the technical aspect of a tool which performs stratification and monetary unit sampling will be described, as well as visual examples that show the results of the implementation and the code.

The first sheet states the instructions for the proper use of the file. As an example of the instruction, the user is advised to not change any of the cell values and to take caution when importing a population into the population sheet.

The second sheet describes the initial parameters needed for the VBA code to function. Parameters such as materiality threshold, clearly trivial threshold, the amount of cells in the population, the amount of cells that will be in the strata, the sample interval and other necessary variables for the VBA code will be defined by the user on this sheet. Assessment of risk and its effect on the confidence level and confidence factors are also stated in this sheet, although they depend entirely on the methodology of the auditing company to define the factors and different risk levels, which is not the goal of this paper.

Figure 1. Initial parameters.

\begin{tabular}{|l|r|}
\hline Materiality threshold & $1.200 .000,00$ \\
\cline { 2 - 2 } Clearly trivial threshold & $48.000,00$ \\
\hline Checksum 1 & 60 \\
\hline Checksum 2 & 61 \\
Checksum 3 (last row of population) & 66 \\
\hline Checksum 4 (stratum) & 35 \\
\hline Checksum 5 (stratum - last row) & 36 \\
\hline
\end{tabular}

\begin{tabular}{|c|c|}
\hline $\begin{array}{c}\text { Choose desired confidence level: } \\
80 \%\end{array}$ & $\begin{array}{c}\text { Confidence factor } \\
\mathbf{1 , 6}\end{array}$ \\
\hline & \\
\hline & $\mathbf{7 5 0 . 0 0 0 , 0 0}$ \\
\hline
\end{tabular}

Source: Author 
The third sheet is the population sheet. In this sheet the user imports the population following the instructions from the first sheet. Once the population has been imported, along with the other data the user has defined on the second sheet where the initial parameters are, the process of stratification may begin.

The fourth sheet is the part of the stratum, which represents values lower than zero. Using the button on this sheet will extract all of the transactions that are lower than zero from the population and present them in the sheet.
The fifth sheet is the part of the stratum, which represents values that cross the materiality threshold. It checks whether each value from the population is higher than the materiality threshold defined in the initial parameters sheet and then extracts that transaction into the sheet.

The sixth sheet is the part of the stratum, which represents values that are clearly trivial. It checks whether the combined value of the lowest transactions is equal to or greater than the sum of the clearly trivial threshold defined in the initial parameters.

Figure 2. Clearly trivial stratum

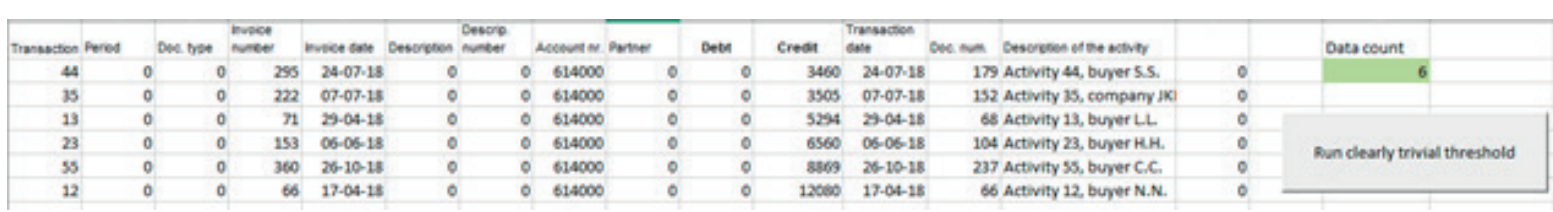

Source: Author

The script takes into consideration all of the values that are lesser than the trivial threshold, sorts them by size and then performs a check whether the sum of the values is larger than or equal to the threshold. If the sum is larger than or equal, it deletes the last (largest) value and performs the check again. If the sum is smaller than the threshold, the script stops and delivers the extracted values.

The seventh sheet is the remaining stratum, and extracts all of the values that are not present in the previous sheets. The button on the sheet performs a check whether the transaction is already present in the aforementioned strata sheets. If a transaction is present, it is automatically skipped and the script moves onto the next transaction, only seeking the transactions that are not present in the previous sheets. Once the script has located all of them, it concludes the stratification process and displays the data for the user.

The eighth and the final sheet performs the monetary unit sampling. This sheet contains the final result, which is based on the remaining stratum from the seventh sheet. Once the script has been ran, it locates the lowest value in the remaining stratum from the previous sheet. The identified value will be the starting value for the calculation of the interval. The highest value for the interval is in the initial parameters sheet.

In the MUS code, the script performs an initial check to see whether the value of the interval is included in the first transaction. If the first transaction is smaller than the value, the script creates a "pool “ where it will store all the values that are below the first interval. Once it finds the transaction, which contains the first interval in it, it checks whether the combined value of the pool and the last transaction that was added in it are larger than the interval, and in that case, subtracts the interval value from the pool. The MUS script repeats the same process until it has located all of the transactions that will form the sample.

After the sample is displayed, the auditor can then examine the sample size and decide whether the sample size was adequate and if MUS was an optimal procedure for the test.

On this sheet, aside from the MUS, other notable information is displayed to the auditor, such as:

- The interval, which is calculated via +Randbetween function using aforementioned variables

- The population value - the sum of the transactions in the remaining stratum

- The sample value - the sum of the transactions in the sample

- The number of transactions in the sample

- Percentage of the population tested - the ratio of the sample value and the population value, and

- The estimated sample size - based on the population value divided by the sampling interval value. 
Figure 3. Monetary Unit Sampling

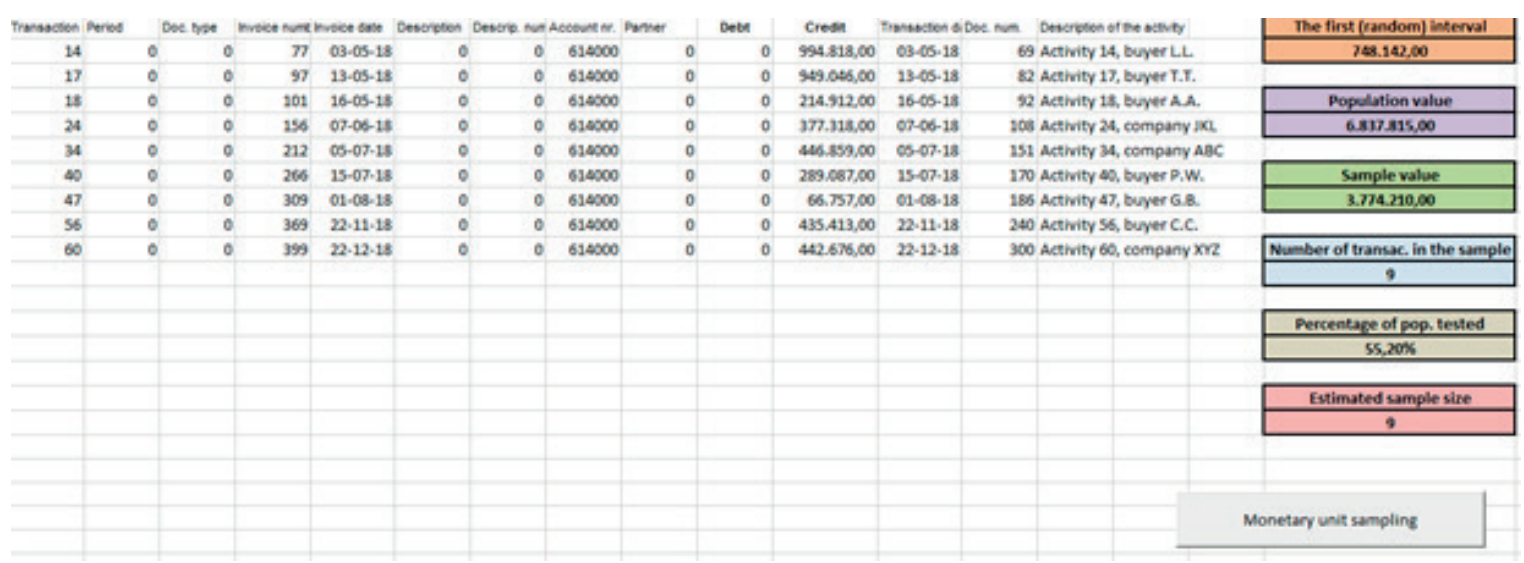

Source: Author

\section{CONCLUSION}

Our findings show that, while it is possible to implement certain procedures and audit processes in complementary software such as Excel, it is necessary for the auditor to weigh the benefits against the costs of creating such a tool. From a practical standpoint, any implementation of an automated sampling method should facilitate the auditing process in general.

From a technical standpoint, building this function into Excel requires the auditor invest time into learning how to code in VBA. While this can be time-consuming, once the auditor has sufficient knowledge of VBA, they can use their skills to implement other features and automate other processes in Excel, or gain better understanding of how Macros work. In general, this is a huge benefit to any auditor.

From a standpoint of quality control, as long as the auditor is prepared to explain the features of the program to quality control and that the results it provides are adequate, the quality control should accept auditors own tools, as there are no official tools advocated by the standards.

Finally, the results from the MUS tool are the same as they would be in any other tool that performs the same function. Stratification and MUS already exist in GAS. However, since small and medium sized audit companies sometimes cannot afford professional software, the results of this research should serve as an incentive for them to build their own functions into the software they use on a regular basis.

\section{LITERATURE}

Lin, C.W., \& Wang, C.H. (2011). A selection model for auditing software. Industrial Management \& Data Systems, Vol. 111 Iss 5 pp. $776-790$

Ahmi, A., \& Kent, S. (2012). The utilisation of generalized audit software (GAS) by external auditors. Managerial Auditing Journal, Vol. 28 Iss 2 pp. 88 - 113

IAASB (2018). ISA 530, Audit sampling. Handbook of international quality control, auditing, review, other assurance, and related services pronouncements, Vol 1 pp. $463-479$.

Wampler, B., \& McEacharn, M. (2005). Monetary-unit sampling using Microsoft Excel. The CPA journal, 75(5), p. 36.

Mijoč I., Mijoč J. (2011). Metode uzoraka u reviziji. Financije, pravo i porezi, $5 / 11$ pp. 109-117

AICPA, (2014). Audit Guide: Audit Sampling First Edition, chapter 6, pp. $95-112$ 